\title{
A STUDY ON BIG DATA FRAMEWORKS AND MACHINE LEARNING TOOL KITS
}

\author{
Imad Sassi and Samir Anter \\ Computer Science Laboratory of Mohammedia (LIM) \\ FST Mohammedia, University of Hassan II, Casablanca, Morocco
}

\begin{abstract}
Big Data is an extremely large amount of structured and unstructured data, gathered from a wide range of sources which often require a fast processing and real time analysis. In this new context, the performances of the traditional techniques are limited. However, to handle these bulky quantities of data, new technologies emerged, called Big Data technologies. In fact, the characteristics of Big Data made the exploration process of these data a painful task. This process is called Big Data Analytics. One of the important challenges of Big Data is to search new technologies or to improve and extend the existing platforms, infrastructures and standard techniques to manage the Big Data. Hadoop / MapReduce paradigm and the Spark framework are among the most prominent solutions for large-scale parallel distributed data processing alongside Machine Learning techniques, in particularly, Deep Learning for performing powerful statistical and predictive analysis. In this paper, we first, give an overview, a classification and a comparison of main Big Data technologies. Then, we focus in particular on Machine Learning platforms and libraries, especially those for Deep Learning. The results show that Spark is a general-purpose computation engine thanks to its very generalized solutions.
\end{abstract}

\section{KEYWORDS}

Big Data, Hadoop, Spark, Machine Learning, Deep Learning

\section{INTRODUCTION}

We currently live in an era marked by the importance of the data. It should be known that in 2019, at every minute, 4 million Google queries sent, 350000 tweets, 7 million snapshots sent on Snapchat, 216 million photos liked on Facebook and 2.4 million photos liked on Instagram and 400 hours of videos uploaded to YouTube. Today, there is more than 4.2 billion internet users who generate and disseminate data. All the signals suggest that the massive production of data is only in its early infancy. Consequently, the advent, since the early 2000s, of the notion of "Big Data" with which multiple challenges, related to the volume, the variety and the velocity, have emerged (Chen et al., 2014). As a result, scientists and companies are now focusing not only on data storage but especially on designing solutions to understand and demystify this huge amount of data to extract value. It is the exploitation of the potential of these data that is promising. Indeed, several Big Data technologies have emerged and their use by large companies, especially the giants of the Web, has become a necessity. These technologies include those that optimize processing time on giant databases such as NoSQL (Han et al., 2011) (Not Only SQL) databases (e.g., MongoDB (Chodorow, 2013), Cassandra (Carpenter, \& Hewitt, 2016)). Also, among these technologies, the server infrastructures make it possible to distribute the processing on thousands of nodes. This is called Massively Parallel Processing. The Hadoop paradigm is undoubtedly the best known of them (White, 2012). It combines the Hadoop Distributed File System (Shvachko et al., 2010), the NoSQL HBase database (George, 2011) and the MapReduce framework (Dean, \& Ghemawat, 2008). Moreover, others Big Data technologies, aimed at moving towards more real-time processing, have emerged (e.g., Storm (Leibiusky et al., 2012), Samza (Noghabi et al., 2017)). Others offer a hybrid system for batch and stream processing such as Apache Spark (Zaharia et al., 2010). There are also complementary technologies for data collection like Kafka (Kreps et al. 2011), data integration like Flume (Hoffman, 2015), coordination and workflow such as Zookeeper (Junqueira, \& Reed, 2013) or the system deployment like Ambari (Wadkar, \& Siddalingaiah, 2014). To process a large amount of data, there are analytical application tools also called Big Data Analytics. One of the most used and certainly the most 
powerful solutions of Big Data Analytics is Machine Learning (Mitchell et al., 1990). In this area, several libraries and platforms are implemented and can be easily integrated into Big Data frameworks. Apache Mahout (Lyubimov, \& Palumbo, 2016), Spark's MLlib (Meng et al., 2016) and Python Scikit-learn (Pedregosa et al., 2011) are examples. Deep Learning (LeCun et al., 2015) is, certainly, one of the most promising Machine Learning techniques, whose software transpositions have produced results that surpass, in a number of use cases, traditional algorithmic approaches. Various tools for Deep Learning, are currently available and are used in different applications areas. Among these, TensorFlow (Abadi et al., 2016), Caffe (Jia et al., 2016) and Deeplearning4j (Team, 2016).

Our main contributions can be summarized as follows: (i) a review and analysis of the main Big Data technologies and the challenges they face; (ii) a classification of its frameworks according to the processing mode (i.e. batch or streaming); (iii) a comparison of these main Big Data frameworks based on several criteria, presenting their advantages and disadvantages. The rest of the paper is organized as follows: In section 4, Big Data Machine Learning tools, mainly those of Deep Learning, are studied and discussed. Sections 5 and 6 conclude the paper and discuss directions for future work.

\section{BIG DATA SYSTEM}

\subsection{Big Data Definition}

The definitions of Big Data are diverse. One of the well-known definitions was proposed by International Data Corporation (IDC), which defines Big Data as follows: "Big Data Technologies describes a new generation of technologies and architectures, designed to economically extract the value from very large volumes of a wide variety of data, allowing a high speed of capture, discovery and / or analysis" (Gantz, \& Reinsel, 2011). It is an extension of Douglas Laney's "3Vs" model, which indicates that challenges and opportunities for growth are three-dimensional data, namely increasing volume, velocity and variety.

\subsection{Big Data Value Chain}

Generally, in a specific Big Data system, there are four phases: data generation, data acquisition, data storage and data analysis. These phases compose what is called a Big Data value chain, as shown in "Figure 1".

\subsubsection{Big Data Generation}

The first phase of the Big Data system is the Big Data generation. It's about how data is generated. There are diverse and complex data generated from various longitudinal and / or distributed data sources.

\subsubsection{Big Data Acquisition}

The second phase of the Big Data system is Big Data acquisition, which includes data collecting, data transmission and data pre-processing (Lyko et al., 2016).

\subsubsection{Big Data Storage}

This phase of the Big Data system includes not only the storage, but also the management of large-scale datasets. In addition to analyzing or interacting with stored data, storage systems must provide several interface, quick query and other programming models (Siddiqa et al., 2017).

\subsubsection{Big Data Analysis}

Big Data analysis is the last and most important step in the Big Data system value chain. Big Data analysis is the process of using analytical tools, algorithms and methods to inspect, transform and model data to uncover hidden potential of Big Data. This is called Big Data Analytics.

To be able to process and analyze Big Data, several frameworks and tools are developed. In the next section, we present the main Big Data technologies. We also present, in the same section, a comparative study of these frameworks (Chintapalli et al., 2016; Gradvohl et al., 2014; Zhang et al., 2017). 


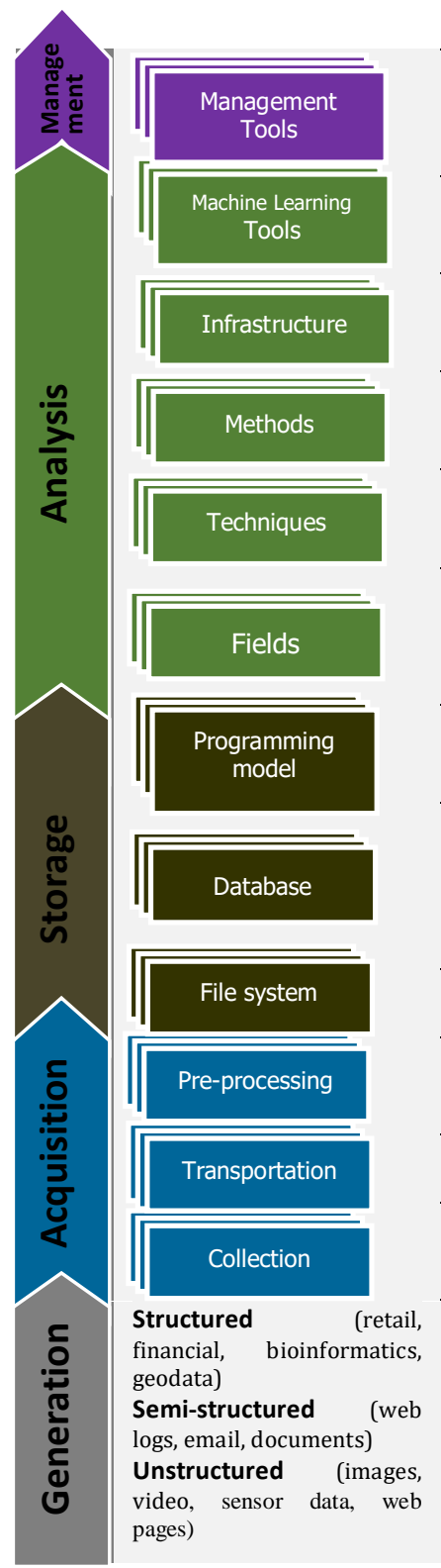

Coordination and Workflow: Zookeeper, Avro and Oozie

System Deployment: Ambari, Whirr, BigTop, Hue

Storage management: HCatalog

Governance and Security: Apache Knox, Apache Falcon

Framework and library: Weka/Pentaho, Apache Mahout, Spark's MLlib, Apache

SystemML Python Scikit-learn, H20, SAMOA, KNMINE, R, MLTK, Vowpal Wabbit, GraphLab, Turi, Orange

Batch only: Apache Hadoop

Stream only: Apache Storm, Apache Samza, Apache S4

Hybrid: Apache Spark, Apache Flink

Bloom Filter, Hashing

Parallel Computing

Indexing

Data Mining, Social Network Analysis, Web Mining

Machine Learning

Visualization Approaches, Optimization Methods

Structured data analysis

Text data analysis, Web data analysis

Multimedia data analysis

Network data analysis, Mobile data analysis

MapReduce

All-Pairs, Pregel

Dryad, GraphLab

Key-value Data Mode (NoSQL): Voldemort, CouchBase, Redis, DataBase, OpenTSDB

Column Data Model (NoSQL): Google BigTable, HBase, Hypertable, Cassandra,

Dynamo, Accumulo

Document Data Model (NoSQL): MongoDB, SimpleDB, Apache CouchDB

Graph Data Model (NoSQL): Aurelius Titan Graph, Neo4J, OrientDB, Giraph

Google File System

Apache HDFS, GlusterFS from Red Hat

Integration: Flume, Kafka, Sqoop

Cleaning: OpenRefine

Redundancy elimination

Inter-DCN transmissions

Intra-DCN Transmissions

Log Files, Sensors

Packets capture, Mobiles devices

Tools: Scribe from Facebook, Kafka from LinkedIn, Chukwa of Hadoop, Timetunnel

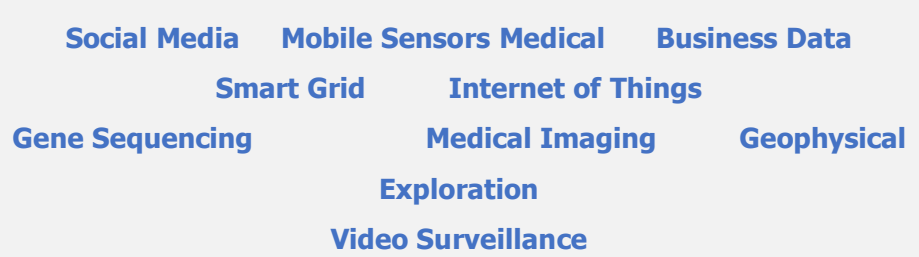

Figure 1. Big Data System value chain

\section{TECHNOLOGIES AND TOOLS FOR BIG DATA ANALYTICS}

\subsection{Stream vs Batch Processing Frameworks}

Depending on the processing time, Big Data analysis can be classified into two paradigms: Stream processing and Batch processing. Stream processing is suitable for applications where the data requires rapid processing and analysis and whose analytical results need to be returned in a very short time. Storm is one of the main frameworks of this processing mode. However, Batch processing is generally used for applications that do not require a high response time. In this processing mode, the data is first stored and then analyzed. MapReduce, actually, is the dominant model of Batch processing.

In the following, we will discuss the main Big Data frameworks for batch and stream processing and we will focus, in particular, on open source frameworks. 


\subsubsection{Hadoop/MapReduce}

MapReduce is a parallel programming model for very large amounts of data processing developed by Google in 2003 with a specific file management system (Google File System) (Ghemawat et al., 2003). The basic strategy of MapReduce is to divide and conquer. The divide and conquer method is implemented in two steps: Map step and Reduce step.

Hadoop is an open source framework developed in 2008 to better generalize the use of massively parallel storage and processing of MapReduce and Google File System. It is widely used to perform Big Data analysis that support large volume of data distributed applications. Hadoop consists of many components and several related projects including YARN (Vavilapalli et al., 2013), Pig, Hive, Sqoop and HBase.

\subsubsection{Dryad}

Dryad is a framework for running applications on multiple servers (Isard et al., 2007). Programs based on this solution can be ported to multiple nodes. It is a clustered platform for large-scale clustering. It is based on dataflow graph processing.

\subsubsection{Spark}

Spark is the next generation paradigm for Big Data processing. Its main goal is to extend Hadoop to a general framework that adopts Resilient Distributed Datasets (RDDs) (Zaharia et al., 2012) in memory computation technique and to offer fault tolerance without replication.

Spark includes seven main components: Spark core of data engine, Spark Cluster Manager (includes Hadoop, Apache Mesos (Hindman et al., 2011) and built-in Standalone cluster manager), Spark SQL, Spark Streaming (Zaharia et al., 2013), Spark Machine Learning Library, Spark GraphX and Spark programming tools.

\subsubsection{Flink}

It is an open source framework that offers batch and stream processing capabilities for distributed Big Data (Carbone et al., 2015). It provides data distribution, communication and fault tolerance for distributed computing.

\subsubsection{Storm}

It is a distributed, scalable and fault-tolerant open source framework designed for stream processing used for real-time analytics, distributed Machine Learning and many other cases (Leibiusky et al., 2012).

\subsubsection{Samza}

It is an open source framework for distributed stream processing. It uses Kafka for messaging and YARN for fault tolerance, processor isolation, security and resource management (Noghabi et al., 2017).

\subsubsection{S4}

S4 is a pluggable, distributed, scalable, fault-tolerant plug-in platform for processing continuous unbounded streaming data (Neumeyer et al., 2010). Since 2011, it has become an Apache Incubator project.

Table 1. summarizes the results of a comparative study presenting the most important characteristics, advantages and disadvantages of the main Big Data frameworks.

\section{BIG DATA MACHINE LEARNING TOOLS}

The major problem of Big Data is not the collection and the storage, but the evaluation of these data. Among the techniques used to extract knowledge from raw data, Machine Learning techniques are the most dominant. It is a branch of Artificial Intelligence that aims to build systems that learn from the received data. Building a system begins by training the machine from a known set of data. Once the system is trained, it must be confronted to the real world and evaluated regularly so that it corrects itself and improves with each new received data. This principle of Machine Learning explains its renewed interest with the appearance of Big Data. 
Table 1. Big Data Frameworks Comparison

\begin{tabular}{|c|c|c|c|c|}
\hline & $\begin{array}{c}\text { Batch vs } \\
\text { Streaming }\end{array}$ & $\begin{array}{c}\text { Characteristics and comparison with } \\
\text { other frameworks }\end{array}$ & Advantages & Disadvantages \\
\hline $\begin{array}{l}\text { Hadoop/ } \\
\text { MapReduce }\end{array}$ & Batch & $\begin{array}{l}\text { efficiency of programming model } \\
\text { (MapReduce) } \\
\text { better in terms of material costs than some } \\
\text { alternatives } \\
\text { Database: HDFS } \\
\text { languages: JAVA, PIG LATIN, HiveQL }\end{array}$ & $\begin{array}{l}\text { scalability -robustness - } \\
\text { fault tolerance -handling of } \\
\text { huge datasets }\end{array}$ & $\begin{array}{l}\text { problem of speed of } \\
\text { execution and processing } \\
\text { - limited SQL support - } \\
\text { inefficient iterative } \\
\text { algorithms }\end{array}$ \\
\hline Spark & $\begin{array}{l}\text { Batch and } \\
\text { Streaming }\end{array} \mid$ & $\begin{array}{l}\text { run a more general directed acyclic graph } \\
\text { (DAG) with more operators than MapReduce } \\
\text {-complete the MapReduce feature with a rich } \\
\text { set of transformations - supports memory } \\
\text { processing unlike MapReduce whose use } \\
\text { storage allows Intermediate data logging - } \\
\text { easy integration with Hadoop ecosystem } \\
\text { tools - exploits main memory unlike Storm - } \\
\text { RDD units are larger than Storm tuples - } \\
\text { more resource consumption than MapReduce }\end{array}$ & 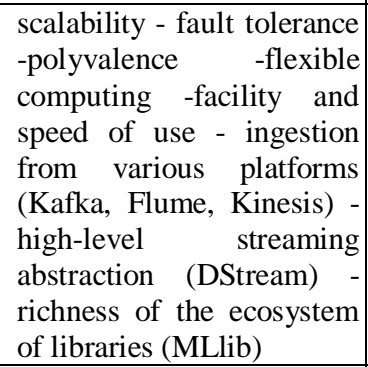 & $\begin{array}{l}\text { Its execution cost more } \\
\text { compared to disk-based } \\
\text { systems }\end{array}$ \\
\hline Flink & $\begin{array}{l}\text { Batch and } \\
\text { Streaming }\end{array} \mid$ & $\begin{array}{l}\text { integrates with YARN and HDFS - unlike } \\
\text { Spark, Flink does not require manual } \\
\text { optimization or adjustment - Unlike Flink, } \\
\text { Spark users can control persistence and } \\
\text { partition scheme on nodes -Flink performs } \\
\text { iterations as cyclic data flows unlike Spark } \\
\text { which executes them by loop unrolling }\end{array}$ & 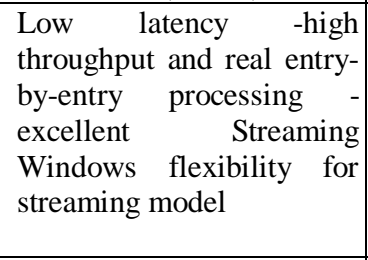 & $\begin{array}{l}\text { remains a young project } \\
\text { that does not have tools } \\
\text { around yet - large scale } \\
\text { deployments are not as } \\
\text { common as other } \\
\text { processing frameworks }\end{array}$ \\
\hline Storm & Streaming & $\begin{array}{l}\text { Unlike Spark, Storm is very difficult to } \\
\text { develop applications with and has limited } \\
\text { resources available in the market - have a } \\
\text { very wide language support more than most } \\
\text { processing frameworks - easily integrates } \\
\text { with existing Big Data technologies }\end{array}$ & $\begin{array}{l}\text { distributed, scalable and } \\
\text { fault-tolerant } \text { processing - } \\
\text { provides rompetitive } \\
\text { performance - real-time } \\
\text { Machine Learning - high } \\
\text { speed and scalability }\end{array}$ & $\begin{array}{l}\text { does not provide message } \\
\text { control guarantees - it is } \\
\text { not suitable for } \\
\text { applications requiring } \\
\text { clusters of several } \\
\text { thousand nodes }\end{array}$ \\
\hline Samza & Streaming & $\begin{array}{l}\text { offers high-level abstractions that are easier } \\
\text { to use than other systems such as Storm - } \\
\text { unlike Storm, it does not have the same } \\
\text { language flexibility - only supports JVM } \\
\text { languages. }\end{array}$ & 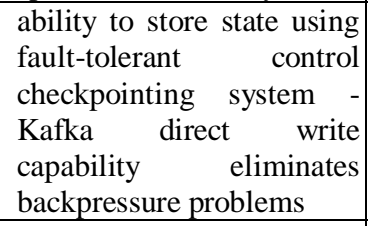 & $\begin{array}{l}\text { use of a Kafka-like } \\
\text { queuing system appears } \\
\text { to be restrictive }- \text { does not } \\
\text { provide accurate } \\
\text { recovery of aggregate } \\
\text { status in failure }\end{array}$ \\
\hline S4 & Streaming & $\begin{array}{l}\text { many competitive properties - automatically } \\
\text { manage communication, scheduling and } \\
\text { distribution across nodes -process continuous } \\
\text { unbounded streams of data }\end{array}$ & $\begin{array}{llr}\text { scalability -robustness } & - \\
\text { fault-tolerant } & - \\
\text { effectiveness in cluster } \\
\text { management }\end{array}$ & $\begin{array}{lr}\text { no support for dynamic } \\
\text { load balancing } & \text {-only } \\
\text { supports partial fault } \\
\text { tolerance }\end{array}$ \\
\hline
\end{tabular}

We present below, the main Big Data Machine Learning projects, including the Deep Learning tools.

\subsection{Machine Learning Tools}

\subsubsection{Mahout}

It is a very rich and complete library of Machine Learning which covers in particular the algorithms of classification and clustering. It distributes its computational algorithms using the Map/Reduce paradigm (Lyubimov, \& Palumbo, 2016).

\subsubsection{Spark MLlib}

This is a scalable Machine Learning library built in Apache Spark designed for high quality algorithms 100 times faster than MapReduce. It offers tools for feature extraction, transformation and basic statistics (Meng et al., 2016). 


\subsubsection{Python Scikit-learn}

It is an open source Machine Learning library. It provides a simple and effective solutions to learning problems. Scikit supports preprocessing, classical algorithms, feature and model selection algorithms (Pedregosa et al., 2011).

\subsubsection{SystemML}

Apache SystemML provides automatic generation of optimized runtime plans ranging from single node computing, in memory, to distributed computations on Hadoop and Spark (Pansare et al., 2018).

\subsubsection{WEKA}

WEKA (Waikato Environment for Knowledge Analysis) is one of the most widely used Java-based Machine Learning software developed at the University of Waikato, New Zealand (Holmes et al., 1994).

\subsubsection{H2O}

$\mathrm{H} 2 \mathrm{O}$ is an open source, in-memory, distributed, fast and scalable predictive analytics and Machine Learning platform (Cook, 2016).

\subsection{Deep Learning Tools}

In this section, we discuss the most popular Deep Learning tools and libraries.

\subsubsection{TensorFlow}

It is a complete Python library. It is made open source by Google in November 2015. It is a set of mathematical functions and calculation methods to develop Machine Learning functions (Abadi et al., 2016).

\subsubsection{Caffe}

It is a Deep Learning library designed to easily express all types of models such as CNN and RNN in a friendly python-based API and run them using an efficient back-end expressed in C++ and CUDA (Jia et al., 2014).

\subsubsection{Deeplearning4j}

DeepLearning4J (DL4J) is a Deep Learning library developed in Java language (and JVM languages) to solve problems involving huge amounts of data in a reasonable amount of time (Team, 2016).

\subsubsection{Torch}

Torch (called Torch7) is an open source Deep Learning library developed by Ronan Collobert. Torch uses C/ $\mathrm{C}++$ libraries as well as CUDA for GPU (Collobert et al., 2002).

\section{DISCUSSION}

With the advent of Big Data, several technologies have emerged, including NoSQL databases and Massively Parallel Processing frameworks. Scientists and companies can choose, depending on their needs, their use case and application requirements, between a vast array of Big Data frameworks for batch processing only, for stream processing only or for a hybrid processing. Hadoop and its MapReduce processing framework is an excellent choice for batch processing model. This one is best suited for handling very large data sets that are not time-sensitive. It offers excellent compatibility and integration with other frameworks and Big Data technologies. In addition, in terms of the cost of the necessary components, Hadoop is inexpensive and efficient for many applications. For stream processing requiring very strict latency, Storm and Samza are a very good choice. Both support many programming languages. Storm is used when unique processing guarantees are needed, it offers competitive performance. Spark's comparison with Storm Streaming shows Spark's performance on Storm. Indeed, Spark is a versatile computation engine thanks to its very general 
solutions. It offers a complete and unified framework because it can be used in batch processing, stream processing, interactive processing as well as iterative processing. This is the right choice for Big Data processing for different data sets of different nature (e.g., text, image, video, graphics) as well as different sources (batch mode or real time). Unlike Storm, which is very difficult to develop with limited resources on the market, Spark is simple, flexible, integrates very well with the Hadoop ecosystem and can primarily handle many types of problems. Spark, offering the possibility to use both modes of processing simultaneously, has many other advantages especially speed, simplicity, scalability, additional modules proposed and easier integration with other tools of the Hadoop ecosystem, but also the possibility of using Machine Learning tools. Apache Spark, is without doubt, a common framework for different types of data processing. For example, real-time data analytics, structured data processing, graph processing, etc. Alongside the explosion of Big Data, several innovations in Machine Learning have emerged, including Mahout, Spark MLlib, Python Scikit-learn and others. Generally, MLlib's dependence on Spark's iterative batch and streaming approaches, as well as its use of in-memory computation, allows tasks to run much faster than those using Mahout.

\section{CONCLUSION AND FUTURE WORK}

The amount of digital data circulating around the world is considerable and will continue to increase in the years to come. The main driver of this growth is the digitization of human life, represented by two main components: connected computing and technology and the Internet of Things. Big Data technologies are fortunately there to help manage the large amounts of data transiting through its ecosystem, but also to explore new data, including those characterized by the speed of the flows it generates. In this work, we studied the major Big Data technologies by referring to the Big Data system value chain. We also evaluated the main batch and stream processing platforms based on several criteria. Much work remains to be done to increase the performance of Big Data technologies to meet the challenges it faces. In this direction, Deep Learning is at the forefront of Machine Learning with great promise. It has demonstrated that it is extremely promising for many areas of Big Data Machine Learning. Cloud computing is also a fertile area of Big Data. It is a highly achievable technology that attracts a large number of researchers to develop it and try to apply it to Big Data issues. From an algorithmic point of view, it's very natural to think about improving or extending the performance of traditional Machine Learning algorithms to make them more suitable for the context of large-scale data or to search for new algorithms that may be good solutions for Big Data Analytics.

\section{REFERENCES}

Abadi, M. et al, 2016. TensorFlow: A System for Large-Scale Machine Learning. In 12th \{USENIX\} Symposium on Operating Systems Design and Implementation (\{OSDI\}16) (pp. 265-283).

Carbone, P. et al, 2015. Apache flink: Stream and batch processing in a single engine. Bulletin of the IEEE Computer Society Technical Committee on Data Engineering, 36(4).

Carpenter, J. et al, 2016. Cassandra: The Definitive Guide: Distributed Data at Web Scale. " O'Reilly Media, Inc.".

Chen, M. et al, 2014. Big data: A survey. Mobile networks and applications, 19(2), 171-209.

Chintapalli, S. et al, 2016. Benchmarking streaming computation engines: storm, flink and spark streaming. In Parallel and Distributed Processing Symposium Workshops, 2016 IEEE International (pp. 1789-1792). IEEE.

Chodorow, K., 2013. MongoDB: The Definitive Guide: Powerful and Scalable Data Storage. " O'Reilly Media, Inc.".

Collobert, R. et al, 2002. Torch: a modular machine learning software library (No. REP_WORK). Idiap.

Cook, D., 2016. Practical machine learning with H2O: powerful, scalable techniques for deep learning and AI. " O'Reilly Media, Inc.".

Dean, J., and Ghemawat, S., 2008. MapReduce: simplified data processing on large clusters. Communications of the ACM, 51(1), 107-113.

Gantz, J., and Reinsel, D., 2011. Extracting value from chaos. IDC iview, 1142(2011), 1-12.

George, L., 2011. HBase: the definitive guide: random access to your planet-size data. " O'Reilly Media, Inc.".

Ghemawat, S. et al, 2003. The Google file system. 
Gradvohl, A. L. S. et al, 2014. Comparing distributed online stream processing systems considering fault tolerance issues. Journal of Emerging Technologies in Web Intelligence, 6(2), 174-179.

Han, J. et al, 2011. Survey on NoSQL database. In 2011 6th international conference on pervasive computing and applications (pp. 363-366). IEEE.

Hindman, B. et al, 2011. Mesos: A platform for fine-grained resource sharing in the data center. In NSDI (Vol. 11, No. 2011, pp. 22-22).

Hoffman, S., 2015. Apache flume: Distributed log collection for hadoop. Packt Publishing Ltd.

Holmes, G. et al, 1994. Weka: A machine learning workbench.

Isard, M. et al, 2007. Dryad: distributed data-parallel programs from sequential building blocks. In ACM SIGOPS operating systems review (Vol. 41, No. 3, pp. 59-72). ACM.

Jia, Y. et al, 2014. Caffe: Convolutional architecture for fast feature embedding. In Proceedings of the 22nd ACM international conference on Multimedia (pp. 675-678). ACM.

Junqueira, F., and Reed, B., 2013. ZooKeeper: distributed process coordination. " O'Reilly Media, Inc.".

Kreps, J. et al, 2011. Kafka: A distributed messaging system for log processing. In Proceedings of the NetDB (pp. 1-7).

LeCun, Y. et al, 2015. Deep learning. nature, 521(7553), 436.

Leibiusky, J. et al, 2012. Getting started with storm. " O'Reilly Media, Inc.".

Lyko, K. et al, 2016. Big data acquisition. In New Horizons for a Data-Driven Economy (pp. 39-61). Springer, Cham.

Lyubimov, D., and Palumbo, A., 2016. Apache Mahout: Beyond MapReduce. CreateSpace Independent Publishing Platform.

Meng, X. et al, 2016. Mllib: Machine learning in apache spark. The Journal of Machine Learning Research, 17(1), 1235-1241.

Mitchell, T. et al, 1990. Machine learning. Annual review of computer science, 4(1), 417-433.

Neumeyer, L. et al, 2010. S4: Distributed stream computing platform. In Data Mining Workshops (ICDMW), 2010 IEEE International Conference on (pp. 170-177). IEEE.

Noghabi, S. A. et al, 2017. Samza: stateful scalable stream processing at LinkedIn. Proceedings of the VLDB Endowment, 10(12), 1634-1645.

Pansare, N. et al, 2018. Deep Learning with Apache SystemML. arXiv preprint arXiv:1802.04647.

Pedregosa, F. et al, 2011. Scikit-learn: Machine learning in Python. Journal of machine learning research, 12(Oct), 2825-2830

Shvachko, K. et al, 2010. The hadoop distributed file system. In MSST (Vol. 10, pp. 1-10).

Siddiqa, A. et al, 2017. Big data storage technologies: a survey. Frontiers of Information Technology \& Electronic Engineering, 18(8), 1040-1070.

Team, D., 2016. Deeplearning4j: Open-source distributed deep learning for the jvm. Apache Software Foundation License, 2.

Vavilapalli, V. K. et al, 2013. Apache hadoop yarn: Yet another resource negotiator. In Proceedings of the 4th annual Symposium on Cloud Computing (p. 5). ACM.

Wadkar, S., and Siddalingaiah, M., 2014. Apache ambari. In Pro Apache Hadoop (pp. 399-401). Apress, Berkeley, CA.

White, T., 2012. Hadoop: The definitive guide. " O'Reilly Media, Inc.".

Zaharia, M. et al, 2010. Spark: Cluster computing with working sets. HotCloud, 10(10-10), 95.

Zaharia, M. et al, 2012. Resilient distributed datasets: A fault-tolerant abstraction for in-memory cluster computing. In Proceedings of the 9th USENIX conference on Networked Systems Design and Implementation (pp. 2-2). USENIX Association.

Zaharia, M. et al, 2013. Discretized streams: Fault-tolerant streaming computation at scale. In Proceedings of the twenty-fourth ACM symposium on operating systems principles (pp. 423-438). ACM.

Zhang, K. et al, 2017. A Comparison of Distributed Machine Learning Platforms. In 2017 26th International Conference on Computer Communication and Networks (ICCCN) (pp. 1-9). IEEE. 